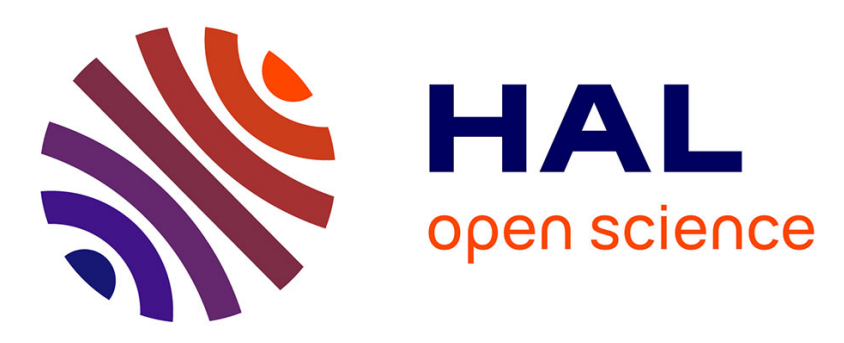

\title{
Identification of MIMO switched state-space models
} Laurent Bako, van Luong Le, Fabien Lauer, Gérard Bloch

\section{To cite this version:}

Laurent Bako, van Luong Le, Fabien Lauer, Gérard Bloch. Identification of MIMO switched statespace models. American Control Conference, ACC 2013, Jun 2013, Washington, United States. pp.CD-ROM. hal-00798991

\section{HAL Id: hal-00798991 https://hal.science/hal-00798991}

Submitted on 11 Mar 2013

HAL is a multi-disciplinary open access archive for the deposit and dissemination of scientific research documents, whether they are published or not. The documents may come from teaching and research institutions in France or abroad, or from public or private research centers.
L'archive ouverte pluridisciplinaire HAL, est destinée au dépôt et à la diffusion de documents scientifiques de niveau recherche, publiés ou non, émanant des établissements d'enseignement et de recherche français ou étrangers, des laboratoires publics ou privés. 


\title{
Identification of MIMO switched state-space models
}

\author{
Laurent Bako ${ }^{1}$, Van Luong $\mathrm{Le}^{2}$, Fabien Lauer ${ }^{3}$ and Gérard Bloch ${ }^{2}$
}

\begin{abstract}
Identifying switched linear models directly from input-output measurements only is known to be a non-trivial identification problem. When switched state-space models are considered in a general setting and both the continuous state and the discrete mode are unmeasured, the problem proves to be a much harder realization problem. The present paper describes a method for identifying discrete-time switched linear state-space models from input-state-output measurements. While the discrete mode is unknown, we assume here that the continuous state is measured along with the input and output signals. Given a finite collection of such measured data, we propose a sparsity-inducing optimization approach for estimating the matrices associated with each submodel.
\end{abstract}

\section{INTRODUCTION}

Switched linear dynamical systems can be described with input-output models or with state-space models. Models of the first type (which are generally of Switched AutoRegressive eXogenous type) express directly the current output as a function of the past outputs and inputs which are all measurable. This feature is suitable from an identification point of view, at least when the system to be identified is SISO. This is probably the reason why the majority of existing works focus on this type of models [1], [2], [3], [4], [5], [6], [7], [8]. Unfortunately, input-output SARX models are not very appropriate for identifying switched MIMO systems. For they typically suffer from a structural identifiability problem; the dimension of the corresponding regressor is overly high, hence preventing in general the data matrix to enjoy a full rank property no matter how exciting the continuous input is. As a consequence, no proper estimation of parameters can be achieved.

MIMO switched systems can be more conveniently described with minimal state-space models which involve explicitly the internal state of the system. Because the state of such a model is of minimal dimension, rank deficiency problems on the data matrices can be avoided provided the continuous input and switching signals are sufficiently exciting. The identification of switched multivariable systems can therefore be made more effective when they are described by minimal state-space models. One more argument in favor of state-space models is that they are the most widely used form of dynamic models in control systems engineering. There are two possible ways to obtain a switched model

\footnotetext{
${ }^{1}$ L. Bako is with (1) Univ Lille Nord de France, F-59000 Lille, France and (2) Mines Douai, IA, F-59500 Douai, France. E-mail: laurent.bako@mines-douai.fr

${ }^{2}$ V. L. Le and G. Bloch are with Université de Lorraine, CRAN CNRS, Nancy, France. E-mails: van-luong.leduniv-lorraine.fr, gerard.blocheuniv-lorraine.fr

${ }^{3} \mathrm{~F}$. Lauer is with Université de Lorraine, LORIA CNRS Inria, Nancy, France. E-mail: fabien.lauer@loria.fr
}

in state-space form from empirical data samples: identify an input-output model and then convert it through realization techniques [9], or identify directly a state-space model [10]. The first option is practical but only for SISO/MISO systems since, as we have already argued, MIMO input-output models of SARX type are difficult to compute. If one assumes that the continuous state is not fully measurable, then the second alternative appears to be a very hard realization problem (see e.g., [10]); it can reasonably work in practice only under some restrictive assumptions (e.g. minimum dwelltime assumption in [11], [12], [13], [14] or known switching times assumption in [14]).

In this paper, we assume that the continuous state is measured and propose an identification method for MIMO switched linear systems. Measurability of the continuous state is not a strong assumption because there are many real situations where the state components correspond to physical, interpretable and measurable variables. Examples of control systems having in general fully measurable states include aircraft, DC-motor, inverted pendulum, etc, which can often be modeled as switched linear/affine systems. Following the paper [5], we propose here a sparse optimization approach to the multivariable identification problem. The identification problem is formulated ideally as the problem of minimizing the number of nonzero components in a vector of normed fitting errors. As such however, the solution is not directly computable at a reasonable cost. We therefore implement a convex relaxation strategy, which is guaranteed, under certain conditions, to find exactly the searched parameter matrices from noise-free data samples. A robust version of the method is then discussed for the case of highly noisy measurements and estimation error bounds are derived.

Section II presents the switched state-space model and describes the identification problem. Section II-A elaborates on the proposed identification method in an ideal noiseless framework. Section II-C deals with some robustness issues and provides bounds on the estimation errors. We conclude the paper in Section IV.

\section{SWITCHED LINEAR STATE-SPACE MODEL IDENTIFICATION}

We consider the discrete-time switched linear system (SLS) represented by

$$
\left\{\begin{aligned}
x(t+1) & =A_{\sigma(t)} x(t)+B_{\sigma(t)} u(t)+w(t), \quad x(0)=x_{0} \\
y(t) & =C_{\sigma(t)} x(t)+D_{\sigma(t)} u(t)+v(t)
\end{aligned}\right.
$$

where $t \in \mathbb{Z}_{+}$is a time index, $x(t) \in \mathbb{R}^{n}$ is the continuous state at time $t, x_{0}$ is the initial continuous state, $u(t) \in \mathbb{R}^{n_{u}}$ 
is the continuous input, $y(t) \in \mathbb{R}^{n_{y}}$ is the continuous output and $\sigma(t) \in \mathbb{S} \triangleq\{1, \ldots, s\}$ is the discrete mode (or discrete state). $\{w(t)\}$ and $\{v(t)\}$ refer to unknown but bounded error sequences.

It is assumed in this paper that the continuous state is measured as well as the input and the output. But the discrete state sequence i.e., the switching signal $\{\sigma(t)\}$, is unknown. Having assumed measurability of the entire continuous state vector $x(t)$, the second equation of (1) may appear useless. One can consider in general that $D_{i}=0$ for any $i \in \mathbb{S}$ and that $C_{i}$ is just used to select some components of $x(t)$. For the sake of generality, we will however assume here that the $(C, D)$-matrices are unknown and are also subject to switchings. Note that the method to be presented is still applicable even if the second equation of (1) was removed. It is also applicable to any switched static measurement model. By introducing the notations

$$
\begin{array}{ll}
M_{i}=\left[\begin{array}{c|c}
A_{i} & B_{i} \\
\hline C_{i} & D_{i}
\end{array}\right], & z(t)=\left[\frac{x(t+1)}{y(t)}\right], \\
\varphi(t)=\left[\frac{x(t)}{u(t)}\right], & e(t)=\left[\frac{w(t)}{v(t)}\right],
\end{array}
$$

Eq. (1) can be rewritten as

$$
z(t)=M_{\sigma(t)} \varphi(t)+e(t)
$$

This is a switched linear multivariable model. The identification problem then consists in inferring the $s$ submodels described by the matrices $M_{i}, i=1, \ldots, s$, from a finite set of measurements $\{\varphi(t), z(t)\}_{t=0}^{N-1}$.

\section{A. Identification method}

The switched identification problem is known to be very challenging because of the strong coupling between the discrete state and the parameter matrices which are both unknown. The existing literature contains a number of methods which can solve the problem more or less efficiently. But those techniques are designed essentially for SISO or MISO type of systems. Application to general MIMO systems such as (2) would require decomposing the system equations into $n+n_{y}$ separate switched equations and applying the mentioned methods to each of them. An obvious inconvenience of such a procedure is that there is no guarantee that the estimated discrete states from the $n+n_{y}$ separate equations will coincide. The switched multivariable equation (2) has instead to be handled jointly.

This paper extends the sparse optimization approach developed in [5] to multivariable systems. To present clearly the main idea of the method, let us assume for the time being that $e(t)=0$ for any $t$. Then by letting $M$ be a parameter matrix candidate, we can observe the following: if $M=M_{i}$ for some $i \in \mathbb{S}$, then we have $z(t)-M \varphi(t)=0$ whenever $\sigma(t)=i$. This means that the vector sequence $\{z(t)-M \varphi(t)\}$ contains at least as many zero vectors as the number of times the subsystem $i$ has been visited by the system. We will say that such a vector sequence is sparse to some degree. Taking advantage of this observation, we formulate the problem of computing one submodel of system
(2) as the problem of finding $M$ to minimize the number of nonzero vectors in the sequence $\{z(t)-M \varphi(t)\}$. This can be written as an $\ell_{0}$ optimization problem in the form

$$
\min _{M}\|\phi(M)\|_{0}
$$

where

$$
\phi(M)=\left[\|z(0)-M \varphi(0)\|_{2}, \ldots,\|z(N-1)-M \varphi(N-1)\|_{2}\right]^{\top}
$$

and $\|\cdot\|_{0}$ refers to the $\ell_{0}$-"norm" which counts the number of nonzero components. Note that $\phi(M) \in \mathbb{R}^{N}$ is a vector constructed by taking the $\ell_{2}$-"norm" of each column of the error matrix $Z-M \Phi$,

$$
\begin{aligned}
& \Phi=\left[\begin{array}{lll}
\varphi(0) & \ldots & \varphi(N-1)
\end{array}\right] \\
& Z=\left[\begin{array}{lll}
z(0) & \ldots & z(N-1)
\end{array}\right] .
\end{aligned}
$$

As is well-known, problem (3) is a hard combinatorial problem for a large $N$. Exact solution of this type of problem is, in general, intractable in practice. It is therefore necessary to turn to an approximate version of (3). A classical way to proceed is to relax the $\ell_{0}$-norm into an $\ell_{1}$-norm. In effect, the $\ell_{1}$-norm is the best convex approximant of the $\ell_{0}$-norm. Applying this idea leads to the following convex optimization problem

$$
\min _{M}\|W \phi(M)\|_{1}=\sum_{t=0}^{N-1} w(t)\|z(t)-M \varphi(t)\|_{2},
$$

where the components of vector $\phi(M)$ have been weighted; $W=\operatorname{diag}(w(0), \ldots, w(N-1))$ is a diagonal matrix with positive entries $w(t) \geq 0$ for any $t$. The objective function in (4) reads as a sum of $\ell_{2}$-norms of the errors. In principle, any other norm could have been used here. However, they cannot all serve correctly our goal, which is to force the entire (not just some of its entries) vector $z(t)-M \varphi(t)$ to zero wherever possible. For example, a sum of $\ell_{1}$-norms objective function would present the risk of producing (after optimization) a matrix $M$ such that in some vectors of the form $z(t)-M \varphi(t)$ (which are actually expected to be completely zero), only some components are equal to zero. This is obviously an undesirable situation in our context and as discussed in [15], [16].

Examine now under what condition solutions to the convex problem (4) lie in the set $\left\{M_{1}, \ldots, M_{s}\right\}$ of the submodels' parameter matrices. For this purpose, we introduce some more notations.

Notations. For any matrix $M$ of appropriate dimensions, define

$$
\mathcal{I}_{0}(M)=\{t: z(t)-M \varphi(t)=0\} .
$$

We also define the matrix norm $\|\cdot\|_{2, \text { col }}$ such that for any matrix $A=\left[\begin{array}{lll}a_{1} & \cdots & a_{m}\end{array}\right] \in \mathbb{R}^{n \times m}$,

$$
\|A\|_{2, \mathrm{col}}=\sum_{i=1}^{m}\left\|a_{i}\right\|_{2},
$$

$\left\|a_{i}\right\|_{2}$ being the euclidean norm of the column vector $a_{i}$.

Lemma 1: If there is a parameter matrix $M_{i}$ of the 
switched system (2) satisfying

$$
\sum_{t \notin \mathcal{I}_{0}\left(M_{i}\right)} w(t)\|\Lambda \varphi(t)\|_{2}<\sum_{t \in \mathcal{I}_{0}\left(M_{i}\right)} w(t)\|\Lambda \varphi(t)\|_{2}
$$

for any nonzero $\Lambda$ in $\mathbb{R}^{\left(n+n_{y}\right) \times\left(n+n_{u}\right)}$, then $M_{i}$ uniquely solves problem (4).

Proof: The proof follows similar steps as in [17]. $M_{i}$ uniquely solves problem (4) if, for any nonzero matrix $\Lambda$ in $\mathbb{R}^{\left(n+n_{y}\right) \times\left(n+n_{u}\right)}$,

$\sum_{t=0}^{N-1} w(t)\|z(t)-M \varphi(t)\|_{2}<\sum_{t=0}^{N-1} w(t)\|z(t)-(M+\Lambda) \varphi(t)\|_{2}$

or, equivalently, if

$$
\begin{gathered}
\sum_{t \notin \mathcal{I}_{0}\left(M_{i}\right)} w(t)\left[\|z(t)-M \varphi(t)\|_{2}-\|z(t)-(M+\Lambda) \varphi(t)\|_{2}\right] \\
<\sum_{t \in \mathcal{I}_{0}\left(M_{i}\right)} w(t)\|\Lambda \varphi(t)\|_{2} .
\end{gathered}
$$

From the triangle inequality property of vector 2-norm, the following identity $\|a\|_{2}-\|a+b\|_{2} \leq\|b\|_{2}$ holds for any two vectors $a$ and $b$ of compatible dimensions. Applying this, we can observe that the left hand side member of the above inequality is smaller than or equal to $\sum_{t \notin \mathcal{I}_{0}\left(M_{i}\right)} w(t)\|\Lambda \varphi(t)\|_{2}$. From this last observation, it follows that for $M_{i}$ to be a solution to problem (4), it is sufficient that (5) holds.

Lemma 1 provides us with a sufficient condition under which one submodel of the switched system (2) can be recovered by convex optimization. Condition (5) suggests two noteworthy facts: (a)-A parameter matrix $M_{i}$ is all the more recoverable by (4) as the set $\mathcal{I}_{0}\left(M_{i}\right)$ is large, i.e. as the number of data generated by submodel $i$ is large; (b)- If we could, by some means, assign large enough weights $w(t)>0$ to the samples indexed by $t \in \mathcal{I}_{0}\left(M_{i}\right)$, and very small weights $w(t)>0$ to those samples indexed by $t \in\{0, \ldots, N-1\} \backslash \mathcal{I}_{0}\left(M_{i}\right)$, then solving problem (4) would very likely yield $M_{i}$. This last implication of condition (5) will be exploited in Section II-B.

In its form (5) however, the sufficient condition is difficult to check in practice. The next theorem gives a stronger but more checkable sufficient condition of recoverability.

Theorem 1: Assume that the weights $w(t)$ are normalized, i.e., $w(0)+\cdots+w(N-1)=1$ and define

$$
\rho^{o}=\sum_{t \in \mathcal{I}_{0}\left(M_{i}\right)} w(t), \quad \eta=\max _{t}\|\varphi(t)\|_{2},
$$

and

$$
r(\Phi)=\max _{\|\Lambda \Phi\|_{2, \text { col }}=1}\|\Lambda\|_{2} .
$$

Then $M_{i}$ is the unique solution to problem (4) if the following condition holds,

$$
\rho^{o}>1-\frac{1}{2 \eta r(\Phi W)} .
$$

Proof: We know from Lemma 1 that condition (5) is a sufficient condition for $M_{i}$ to solve (uniquely) problem (4).
Note that (5) can be recast in the form

$$
2 \sum_{t \notin \mathcal{I}_{0}\left(M_{i}\right)} w(t)\|\Lambda \varphi(t)\|_{2}<\sum_{t=0}^{N-1} w(t)\|\Lambda \varphi(t)\|_{2}
$$

or, more compactly, in the form

$$
\max _{\|\Lambda \Phi W\|_{2, \mathrm{col}}=1}\left[\sum_{t \notin \mathcal{I}_{0}\left(M_{i}\right)} w(t)\|\Lambda \varphi(t)\|_{2}\right]<1 / 2 .
$$

Denoting $\eta=\max _{t}\|\varphi(t)\|_{2}$ and using the inequality $w(t)\|\Lambda \varphi(t)\|_{2} \leq \eta w(t)\|\Lambda\|_{2}$, it can be seen that the maximum above is bounded from above by $\eta\left(1-\rho^{o}\right) r(\Phi W)$. Therefore $M_{i}$ is the unique solution to (4) if

$$
\eta\left(1-\rho^{o}\right) r(\Phi W)<1 / 2,
$$

which is precisely the claim of the theorem.

The number $r(\Phi)$ defined in (6) can be interpreted as a certain measure of the genericity (or the diversity) of the data matrix $\Phi \cdot r(\Phi)$ is all the smaller as the data in $\Phi$ are more generic. Because $r(\Phi)$ might be a bit difficult to compute directly in the form (6), we give some gross estimates of it in the following.

Lemma 2: $r(\Phi)$ obeys the following properties :

(i) $1 /\|\Phi\|_{2, \text { col }} \leq r(\Phi) \leq\left\|\Phi^{\top}\left(\Phi \Phi^{\top}\right)^{-1}\right\|_{2}$ under the assumption that $\operatorname{rank}(\Phi)=n+n_{u}$.

(ii) $r$ is a decreasing function of the number of data samples (i.e., the number of columns of matrix $\Phi$ ).

(iii) $r(\alpha \Phi)=1 / \alpha r(\Phi)$ for any $\alpha \in \mathbb{R}_{+}$.

Proof: The proofs of (ii) and (iii) follow immediately from the definition of $r(\cdot)$. Therefore we will prove only (i) in details. Observe first that

$$
\|\Lambda \Phi\|_{2, \text { col }}=\sum_{t=0}^{N-1}\|\Lambda \varphi(t)\|_{2} \leq\|\Lambda\|_{2}\|\Phi\|_{2, \text { col }} .
$$

By taking the inverse and multiplying by $\|\Lambda\|_{2}$, we can write

$$
1 /\|\Phi\|_{2, \mathrm{col}} \leq\|\Lambda\|_{2} /\|\Lambda \Phi\|_{2, \mathrm{col}} \leq \max _{\Lambda \neq 0} \frac{\|\Lambda\|_{2}}{\|\Lambda \Phi\|_{2, \mathrm{col}}} .
$$

But in fact

$$
r(\Phi)=\max _{\Lambda \neq 0} \frac{\|\Lambda\|_{2}}{\|\Lambda \Phi\|_{2, \mathrm{col}}}
$$

and the first inequality in (i) follows.

We now prove the second inequality of (i). From the definition (6), pose $\Delta=\Lambda \Phi$, which implies that $\Lambda=$ $\Delta \Phi^{\top}\left(\Phi \Phi^{\top}\right)^{-1}$ since $\Phi$ is assumed to have full row rank. Then by the submultiplicativity property of the matrix 2 norm,

$$
\begin{aligned}
r(\Phi) & =\max _{\|\Delta\|_{2, \text { col }}=1}\left\|\Delta \Phi^{\top}\left(\Phi \Phi^{\top}\right)^{-1}\right\|_{2} \\
& \leq\left\|\Phi^{\top}\left(\Phi \Phi^{\top}\right)^{-1}\right\|_{2} \max _{\|\Delta\|_{2, \text { col }}=1}\|\Delta\|_{2} .
\end{aligned}
$$

To finalize the proof, it remains now to show that

$$
\max _{\|\Delta\|_{2, \mathrm{col}}=1}\|\Delta\|_{2}=1
$$

For this purpose, express $\Delta$ in terms of its columns $\delta_{i} \in$ 
$\mathbb{R}^{n+n_{y}}$ as $\Delta=\left[\begin{array}{lll}\delta_{1} & \cdots & \delta_{N}\end{array}\right]$. Let $\sigma_{1} \geq \cdots \geq \sigma_{n+n_{y}}$ be the singular values of $\Delta^{\top}$, organized in a descending order. Then by using the notation $\|\cdot\|_{F}$ for the Frobenius matrix norm, it is easy to see that

$$
\begin{aligned}
\|\Delta\|_{F}=\sqrt{\left\|\delta_{1}\right\|_{2}^{2}+\cdots+\left\|\delta_{N}\right\|_{2}^{2}} & \leq\left\|\delta_{1}\right\|_{2}+\cdots+\left\|\delta_{N}\right\|_{2} \\
& =\|\Delta\|_{2, \text { col }}
\end{aligned}
$$

Hence the inequality $\|\Delta\|_{F} \leq\|\Delta\|_{2, \text { col }}$ holds true. On the other hand, we know from basic linear algebra that $\|\Delta\|_{2}=$ $\sigma_{1}$ and $\|\Delta\|_{F}=\sqrt{\sigma_{1}^{2}+\cdots+\sigma_{n+n_{y}}^{2}}$. As a consequence, $\|\Delta\|_{2} \leq\|\Delta\|_{F}$. Therefore $\|\Delta\|_{2} \leq\|\Delta\|_{2, \text { col }}$ by transitivity. Fixing $\|\Delta\|_{2, \text { col }}=1$ implies $\|\Delta\|_{2} \leq 1$. In fact this unit upper bound on the 2-norm of $\Delta$ is attainable. To see this, pick for example a matrix $\Delta$ whose columns are all equal to zero except one column whose 2 -norm is set to 1 . Such a matrix realizes $\|\Delta\|_{2, \text { col }}=\|\Delta\|_{2}=1$.

By default the weights are set to $w(t)=1 / N, t=$ $0, \ldots, N-1$, in which case $W=1 / N I_{N}$, with $I_{N}$ denoting the identity matrix. Then $\rho^{o}=\left|\mathcal{I}_{0}\left(M_{i}\right)\right| / N$, with $\left|\mathcal{I}_{0}\left(M_{i}\right)\right|$ denoting the cardinality of the set $\mathcal{I}_{0}\left(M_{i}\right)$, and $r(\Phi W)=N r(\Phi)$. Plugging these expressions into (7) yields the following special case of Theorem 1.

Corollary 1: If

$$
\left|\mathcal{I}_{0}\left(M_{i}\right)\right|>N-\frac{1}{2 \eta r(\Phi)}
$$

then $M_{i}$ is the unique solution to problem (4).

In words, the corollary says that if there is one submodel having generated a number of data samples larger than a certain threshold (depending on the degree of genericity of the data), then this submodel can be computed efficiently by solving problem (4). The good news is that this latter problem is convex and can hence be numerically solved by many well-documented solvers, see e.g. [18].

\section{B. Implementation aspects}

To estimate the parameter matrices, we follow a similar procedure as in [5]. According to Corollary 1, if there is a submodel $i$ which has generated a sufficiently large number of data, the associated parameter matrix $M_{i}$ can be computed by solving the convex problem (4) with $W=1 / N I_{N}$. If none of the submodels has the absolute majority of the data (e.g. in the sense of condition (9)), the solution of problem (4) may not be in $\left\{M_{1}, \ldots, M_{s}\right\}$. To deal with these situations, we can solve a sequence of problems of the type (4) with different weights computed iteratively [19], [5]. The iterative scheme can be defined for a fixed number $r_{\max }$ of iterations as follows. At iteration $r=0, \ldots, r_{\max }$, compute

$$
M^{(r)}=\underset{M}{\arg \min } \sum_{t=0}^{N-1} w^{(r)}(t)\|z(t)-M \varphi(t)\|_{2},
$$

with weights defined, for all $t$, by $w^{(0)}(t)=1 / N$, and

$$
w^{(r)}(t)=\frac{w_{0}^{(r)}(t)}{\sum_{t=0}^{N-1} w_{0}^{(r)}(t)}, \quad \text { if } r \geq 1
$$

where

$$
w_{0}^{(r)}(t)=\frac{1}{\left\|z(t)-M^{(r-1)} \varphi(t)\right\|_{2}+\zeta},
$$

$\zeta>0$ is a small number and $r$ is the iteration number. Since we are dealing here with a sequence of convex optimization problems, they can be numerically implemented using any convex solver. In particular the CVX software package [18] solves efficiently this category of problems in a Matlab environment.

As argued before, the algorithm is expected to converge to one parameter matrix lying in $\left\{M_{1}, \ldots, M_{s}\right\}$. After a first submodel is identified, we need to identify the other submodels. For this purpose, we can remove the data samples pertaining to the already identified submodel and repeat the iterative algorithm over the remaining set of data. We refer to [5] for more details on this aspect.

\section{Robust formulation in the presence of noise}

So far, in the derivation of the method, we have not dealt explicitly with potential noise in the process or in the measurements. Still, as will be shown in Section III, the derived method can stand noise to some extent. However, when the noise level is high, we might need to resort to a more robust implementation. For example, the $\ell_{0}$ problem (3) can be reformulated as

$$
\begin{aligned}
& \min _{M \in \mathbb{R}^{\left(n+n_{y}\right) \times\left(n+n_{u}\right)}, \xi \in \mathbb{R}_{+}^{N}}\|\xi\|_{0} \\
& \text { s.t. }\|z(t)-M \varphi(t)\|_{2} \leq \varepsilon+\xi(t), t=0, \ldots, N-1,
\end{aligned}
$$

where the optimization is performed over the set of parameters $M$ and a set of positive slack variables $\xi$, and where $\varepsilon \geq 0$ is a fixed error threshold. Then, the $\ell_{1}$ relaxation takes the form

$$
\begin{aligned}
& \min _{M \in \mathbb{R}^{\left(n+n_{y}\right) \times\left(n+n_{u}\right)}, \xi \in \mathbb{R}_{+}^{N}}\|W \xi\|_{1} \\
& \text { s.t. }\|z(t)-M \varphi(t)\|_{2} \leq \varepsilon+\xi(t), t=0, \ldots, N-1 .
\end{aligned}
$$

The rationale behind this formulation is that, if $M$ is a parameter matrix representing one submodel $i$, then the errors $\|z(t)-M \varphi(t)\|_{2}$ should be less than a certain threshold $\varepsilon$ whenever $\sigma(t)=i$. Ideally, the threshold must be set to $\max _{t}\|e(t)\|_{2}$. But since this maximum is unknown in general, it may sometimes be preferable to have to tune a regularization parameter instead. Therefore, an alternative formulation is for example

$$
\begin{aligned}
& \min _{M \in \mathbb{R}^{\left(n+n_{y}\right) \times\left(n+n_{u}\right)}, \xi \in \mathbb{R}_{+}^{N}, \varepsilon \in \mathbb{R}_{+}}\|W \xi\|_{1}+\nu \varepsilon \\
& \text { s.t. }\|z(t)-M \varphi(t)\|_{2} \leq \varepsilon+\xi(t), t=0, \ldots, N-1,
\end{aligned}
$$

with $\nu>0$ denoting the regularization parameter which is intended for controlling the tolerance to noise. Large values of $\nu$ tend to tolerate only a moderate amount of noise (or discrepancy). Conversely, relatively small values of $\nu$ should be used in case of highly perturbed measurements. Note that a similar robust implementation has been discussed in the context of nonlinear switched system identification in [20]. We now analyze the robust estimation scheme. 
Lemma 3: Assume $W=I_{N}$ in (11). Denote by $M^{\star}$ a (any) solution to problem (11) and define $\mathcal{I}_{\varepsilon}\left(M_{i}\right)=\{t$ : $\left.\left\|z(t)-M_{i} \varphi(t)\right\|_{2} \leq \varepsilon\right\}$. Then

$\sum_{t \in \mathcal{I}_{\varepsilon}\left(M_{i}\right)}\left\|\left(M^{\star}-M_{i}\right) \varphi(t)\right\|_{2} \leq 2\left|\mathcal{I}_{\varepsilon}\left(M_{i}\right)\right| \varepsilon+\sum_{t \in \mathcal{I}_{\varepsilon}^{c}\left(M_{i}\right)}\left\|\left(M^{\star}-M_{i}\right) \varphi(t)\right\|_{2}$,

where $\mathcal{I}_{\varepsilon}^{c}\left(M_{i}\right)$ is the complement of $\mathcal{I}_{\varepsilon}\left(M_{i}\right)$ in the set of indices $\{0,1, \ldots, N-1\}$.

Proof: We start by observing that problem (11) is, when the variable $\xi$ is removed, equivalent to

$$
\min _{M} \sum_{t=0}^{N-1} \max \left[0,\|z(t)-M \varphi(t)\|_{2}-\varepsilon\right]
$$

It then follows that

$$
\begin{aligned}
& \sum_{t=0}^{N-1} \max \left(0,\left\|z(t)-M^{\star} \varphi(t)\right\|_{2}-\varepsilon\right) \leq \\
& \sum_{t=0}^{N-1} \max \left(0,\left\|z(t)-M_{i} \varphi(t)\right\|_{2}-\varepsilon\right)
\end{aligned}
$$

Noting that $\max \left(0,\left\|z(t)-M_{i} \varphi(t)\right\|_{2}-\varepsilon\right)=0$ for any $t \in$ $\mathcal{I}_{\varepsilon}\left(M_{i}\right)$, the previous inequality can be written as

$$
\begin{aligned}
\sum_{t \in \mathcal{I}_{\varepsilon}\left(M_{i}\right)} & \max \left(0,\left\|z(t)-M^{\star} \varphi(t)\right\|_{2}-\varepsilon\right) \leq \\
& \sum_{t \in \mathcal{I}_{\varepsilon}^{c}\left(M_{i}\right)} \max \left(0,\left\|z(t)-M_{i} \varphi(t)\right\|_{2}-\varepsilon\right) \\
& -\sum_{t \in \mathcal{I}_{\varepsilon}^{c}\left(M_{i}\right)} \max \left(0,\left\|z(t)-M^{\star} \varphi(t)\right\|_{2}-\varepsilon\right) .
\end{aligned}
$$

We will now find an underestimate of the term on the lefthand side of (14) and an overestimate for the right-hand side member. Pose $H_{i}=M^{\star}-M_{i}$. For $t$ in $\mathcal{I}_{\varepsilon}\left(M_{i}\right)$,

$$
\begin{aligned}
\left\|H_{i} \varphi(t)\right\|_{2} & =\left\|\left(M^{\star} \varphi(t)-z(t)\right)+\left(z(t)-M_{i} \varphi(t)\right)\right\|_{2} \\
& \leq\left\|z(t)-M^{\star} \varphi(t)\right\|_{2}+\varepsilon
\end{aligned}
$$

which implies that

$$
\left\|H_{i} \varphi(t)\right\|_{2}-2 \varepsilon \leq \max \left(0,\left\|z(t)-M^{\star} \varphi(t)\right\|_{2}-\varepsilon\right) .
$$

Therefore, $\sum_{t \in \mathcal{I}_{\varepsilon}\left(M_{i}\right)}\left\|H_{i} \varphi(t)\right\|_{2}-2\left|\mathcal{I}_{\varepsilon}\left(M_{i}\right)\right| \varepsilon$ is an underestimate. It remains to find the overestimate. To this end, apply the identity $\max (0, a)=1 / 2(a+|a|)$. Then the righthand side part of (14) equals

$$
\begin{aligned}
1 / 2 \sum_{t \in \mathcal{I}_{\varepsilon}^{c}\left(M_{i}\right)}\left[\left\|z(t)-M_{i} \varphi(t)\right\|_{2}-\left\|z(t)-M^{\star} \varphi(t)\right\|_{2}\right]+ \\
1 / 2 \sum_{t \in \mathcal{I}_{\varepsilon}^{c}\left(M_{i}\right)}\left[\left|\left\|z(t)-M_{i} \varphi(t)\right\|_{2}-\varepsilon\right|\right. \\
\left.-\left|\left\|z(t)-M^{\star} \varphi(t)\right\|_{2}-\varepsilon\right|\right]
\end{aligned}
$$

Recall now that for any real numbers $a, b$, and for any vectors $c, d$, the following holds, $|a|-|b| \leq|a-b|$ and $\|c\|_{2}-$ $\|d\|_{2} \leq\|c-d\|_{2}$. Using these identities in (16) yields

$$
(16) \leq \sum_{t \in \mathcal{I}_{\varepsilon}^{c}\left(M_{i}\right)}\left\|H_{i} \varphi(t)\right\|_{2} .
$$

Recapitulating,

$$
\sum_{t \in \mathcal{I}_{\varepsilon}\left(M_{i}\right)}\left\|H_{i} \varphi(t)\right\|_{2}-2\left|\mathcal{I}_{\varepsilon}\left(M_{i}\right)\right| \varepsilon \leq \sum_{t \in \mathcal{I}_{\varepsilon}^{c}\left(M_{i}\right)}\left\|H_{i} \varphi(t)\right\|_{2}
$$

which is indeed the sought result.

Theorem 2: Assume $W=I_{N}$ in (11) and let the notations of Lemma 3 remain in force. Then, for any parameter matrix $M_{i}$ satisfying

$$
\left|\mathcal{I}_{\varepsilon}\left(M_{i}\right)\right|>N-\frac{\sigma_{\min }}{2 \eta}
$$

with $\sigma_{\min }$ being the smallest singular value of $\Phi^{\top}$ (which is nonzero if $\Phi$ is full row rank), the estimation error is bounded as follows

$$
\left\|M^{\star}-M_{i}\right\|_{2} \leq \frac{\varepsilon}{\eta\left[1-\frac{N-\frac{\sigma_{\min }}{2 \eta}}{\left|\mathcal{I}_{\varepsilon}\left(M_{i}\right)\right|}\right]}
$$

Proof: The theorem is a direct consequence of the relation (12). In effect, (12) reads as

$$
\begin{aligned}
\left\|H_{i} \Phi\right\|_{2, \text { col }} & =\sum_{t=0}^{N-1}\left\|H_{i} \varphi(t)\right\|_{2} \\
& \leq 2\left|\mathcal{I}_{\varepsilon}\left(M_{i}\right)\right| \varepsilon+2 \sum_{t \in \mathcal{I}_{\varepsilon}^{c}\left(M_{i}\right)}\left\|H_{i} \varphi(t)\right\|_{2}
\end{aligned}
$$

where $H_{i}=M^{\star}-M_{i}$. We have already seen in the proof of Lemma 2 that $\left\|H_{i} \Phi\right\|_{2} \leq\left\|H_{i} \Phi\right\|_{2, \text { col }}$. On the other hand it can be easily established that $\sigma_{\min }\left\|H_{i}\right\|_{2} \leq\left\|H_{i} \Phi\right\|_{2}$. By combining these two remarks with the fact that $\left\|H_{i} \varphi(t)\right\|_{2} \leq$ $\left\|H_{i}\right\|_{2} \eta$, we can write

$$
\sigma_{\min }\left\|H_{i}\right\|_{2} \leq 2 \varepsilon\left|\mathcal{I}_{\varepsilon}\left(M_{i}\right)\right|+2 \eta\left\|H_{i}\right\|_{2}\left(N-\left|\mathcal{I}_{\varepsilon}\left(M_{i}\right)\right|\right) .
$$

Manipulating a little this last inequality leads to (19).

Theorem 2 constitutes an interesting stability result. If for a given $\varepsilon$ there is a parameter matrix $M_{i}$ that fulfills condition (18), then the theorem provides an explicit bound (which is proportional to the design parameter $\varepsilon$ ) on the estimation error for $M_{i}$. If, on the contrary, $\varepsilon$ is such that none of the submodels satisfies (18), not much can be said about the bound on the deviation of $M^{\star}$ from the true parameter matrices. Finally, Corollary 1 appears to be a special case of Theorem 2 when the data are noise-free and $\varepsilon=0$.

\section{NUMERICAL EXPERIMENTS}

To evaluate the proposition of the paper, we consider an example of switched linear system having 2 inputs, 2 outputs and 3 submodels whose state dimension is equal to 3 . The system has the form (1) and its matrices are given as

$$
\left\{\begin{array}{rlr}
A_{1}=\left[\begin{array}{ccc}
0.15 & 0.40 & -0.65 \\
-0.75 & 0.1 & -0.35 \\
0.20 & 0.70 & 0.20
\end{array}\right], & B_{1}=\left[\begin{array}{cc}
-0.20 & 0.45 \\
-0.06 & 0 \\
0.22 & 0
\end{array}\right] \\
C_{1}=\left[\begin{array}{ccc}
0 & 0.40 & 0.45 \\
-1 & -0.60 & 0.90
\end{array}\right], & D_{1}=\left[\begin{array}{cc}
0.35 \\
-1.70 & -0.25
\end{array}\right]
\end{array}\right.
$$




$$
\begin{array}{rlrl}
A_{2} & =\left[\begin{array}{ccc}
0.27 & 0.24 & -0.55 \\
0.24 & 0.65 & 0.30 \\
-0.55 & 0.30 & 0.27
\end{array}\right], & B_{2}=\left[\begin{array}{cc}
-0.55 & 0 \\
-1.40 & 1 \\
0.05 & -0.72
\end{array}\right] \\
C_{2}=\left[\begin{array}{ccc}
0.70 & 1 & -0.27 \\
-0.35 & 0 & -1.10
\end{array}\right], & D_{2}=\left[\begin{array}{cc}
2.15 & 0.25 \\
0 & -0.36
\end{array}\right] \\
\left\{\begin{array}{lll}
A_{3} & =\left[\begin{array}{ccc}
0.45 & 0.02 & 0.42 \\
-0.17 & 0.53 & 0.20 \\
0.38 & 0.26 & -0.66
\end{array}\right], & B_{3}=\left[\begin{array}{cc}
0 & 0.15 \\
0.27 & -0.46 \\
0.07 & 0.54
\end{array}\right] \\
C_{3}=\left[\begin{array}{lll}
0 & 0.60 & 0.28 \\
0 & 0.86 & 0.45
\end{array}\right], & D_{3}=0.90 \\
0 & 0.85
\end{array}\right]
\end{array}
$$

We run 100 simulations to generate 100 different sets of 1000 input-state-output data each. For each simulation, the data are generated with a normally distributed input, $u(t) \sim$ $\mathcal{N}\left(0, I_{2}\right)$. The discrete state $\sigma(t)$ is uniformly distributed in $\mathbb{S}=\{1,2,3\}$ so that all the three modes have approximately the same number of data samples. The process noise $\{w(t)\}$ and measurement noise $\{v(t)\}$ are both normally distributed. They are sampled, for each simulation, so as to achieve a moderate Signal-to-Noise-Ratio (SNR) of $30 \mathrm{~dB}$ of respectively the state and the output. A consequence of the noise being sampled from a Gaussian distribution is that the corresponding sequence $\{e(t)\}$ in (2) is not absolutely bounded. We apply the non-robust version of the algorithm as described in Section II-B to the obtained 100 datasets separately. By repeating the experiment for different levels of noise, we obtain some average estimation errors for each parameter matrix as reported in Table I. These results show that the proposed method has the potential of identifying correctly a switched linear system of the form (1).

TABLE I

AVerage Relative normed ERrors $\left\|M_{i}-\hat{M}_{i}\right\|_{\mathrm{F}} /\left\|M_{i}\right\|_{\mathrm{F}}$ OVER 100 INDEPENDENT RUNS.

\begin{tabular}{|c|ccc|}
\hline$i$ & 1 & 2 & 3 \\
\hline $30 \mathrm{~dB}$ & 0.0081 & 0.0062 & 0.0109 \\
\hline $25 \mathrm{~dB}$ & 0.0377 & 0.0344 & 0.0357 \\
\hline $20 \mathrm{~dB}$ & 0.0782 & 0.0610 & 0.0770 \\
\hline $15 \mathrm{~dB}$ & 0.1614 & 0.1081 & 0.1536 \\
\hline
\end{tabular}

\section{CONCLUSION}

In this paper we generalized the sparse optimization approach to the identification of MIMO switched linear state-space systems where the continuous state is assumed available. We view the problem of estimating one submodel of the switched system as the problem of minimizing the number of nonzero entries in a vector of normed errors. Because this is an NP-hard problem, we relax this first formulation and show that the different submodels can be recovered through convex optimization. Some conditions of exact recovery from noiseless data are also discussed. For the noisy case, an alternative robust formulation of the identification problem is proposed. With respect to this latter formulation, we derived an explicit bound on the estimation error.

In this work, we have assumed that the continuous state is fully measurable, which can be restrictive. To broaden the scope of applicability of the proposed identification method, it is desirable to be able to drop this assumption. This is a challenging problem which, hopefully, will be solved in future research.

\section{REFERENCES}

[1] R. Vidal, S. Soatto, Y. Ma, and S. Sastry, "An algebraic geometric approach to the identification of a class of linear hybrid systems," in Conference on Decision and Control, Maui, Hawaii, USA, 2003.

[2] R. Vidal, "Recursive identification of switched ARX systems," Automatica, vol. 44, pp. 2274-2287, 2008.

[3] A. Bemporad, A. Garulli, S. Paoletti, and A. Vicino, "A bounded-error approach to piecewise affine system identification," IEEE Transactions on Automatic Control, vol. 50, pp. 1567-1580, 2005.

[4] F. Lauer, G. Bloch, and R. Vidal, "A continuous optimization framework for hybrid system identification," Automatica, vol. 47, pp. 608613, 2011.

[5] L. Bako, "Identification of switched linear systems via sparse optimization," Automatica, vol. 47, pp. 668-677, 2011.

[6] N. Ozay, M. Sznaier, C. Lagoa, and O. Camps, "A sparsification approach to set membership identification of a class of affine hybrid systems," IEEE Transactions on Automatic Control, vol. 57, pp. 634648, 2012.

[7] S. Paoletti, A. Juloski, G. Ferrari-Trecate, and R. Vidal., "Identification of hybrid systems: A tutorial.," European Journal of Control, vol. 13, pp. 242-260, 2007.

[8] L. Bako and R. Vidal, "Algebraic identification of switched MIMO ARX models," in Hybrid Systems: Control and Computation (M. Egerstedt and B. Mishra, eds.), vol. 4981 of LCNS, pp. 43-57, Springer Verlag, 2008.

[9] M. Petreczky, L. Bako, and J. H. van Schuppen, "Identifiability of discrete-time linear switched systems," in Hybrid Systems: Computation and Control, Stockholm, Sweden, 2010.

[10] L. Bako, G. Mercère, R. Vidal, and S. Lecoeuche., "Identification of switched linear state space models without minimum dwell time," in IFAC Symposium on System Identification, Saint Malo, France, 2009.

[11] K. Huang, A. Wagner, and Y. Ma, "Identification of hybrid linear time-invariant systems via subspace embedding and segmentation," in Conference on Decision and Control, Atlantis, Paradise Island, Bahamas, 2004.

[12] J. Borges, V. Verdult, M. Verhaegen, and M. A. Botto, "A switching detection method based on projected subspace classification," in Conference on Decision and Control-European Control Conference, Seville, Spain, 2005.

[13] L. Bako, G. Mercère, and S. Lecoeuche, "Online structured subspace identification with application to switched linear systems," International Journal of Control, vol. 82, pp. 1496-1515, 2009.

[14] V. Verdult and M. Verhaegen, "Subspace identification of piecewise linear systems," in Conference on Decision and Control, Atlantis, Paradise Island, Bahamas, 2004.

[15] H. Ohlsson, L. Ljung, and S. Boyd, "Segmentation of ARX-models using sum-of-norms regularization," Automatica, vol. 46, pp. 1107$1111,2010$.

[16] D. Chen, L. Bako, and S. Lecoeuche, "A recursive sparse learning method: Application to jump markov linear systems," in 18th IFAC World Congress, Milano, Italy, 2011.

[17] L. Bako, K. Boukharouba, and S. Lecoeuche, "Recovering piecewise affine maps by sparse optimization.," in IFAC Symposium on System Identification, Brussels, Belgium, 2012.

[18] M. Grant and S. Boyd, "CVX: Matlab software for disciplined convex programming, version 1.2 (june 2009, build 711).".

[19] E. J. Candès, M. Wakin, and S. Boyd, "Enhancing sparsity by reweighted $\ell_{1}$ minimization," Journal Fourier Analysis and Applications, vol. 14, pp. 877-905, 2008.

[20] V. L. Le, F. Lauer, L. Bako, and G. Bloch, "Learning nonlinear hybrid systems: from sparse optimization to support vector regression," in ACM Conference on Hybrid Systems: Computation and Control, Philadelphia, PA, USA, 2013. 